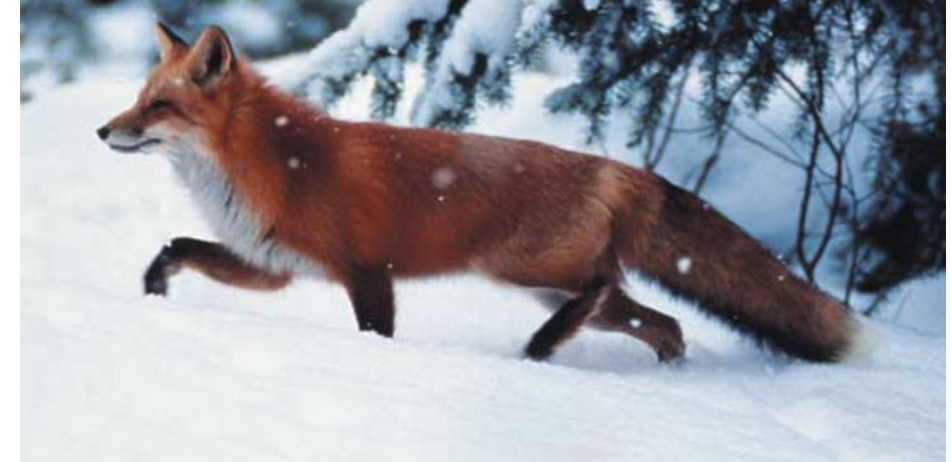

TUMORIGENESIS

\title{
OutFOXing liver cancer
}

Targeted therapies for

hepatocellular carcinoma (HCC) are difficult to develop, as the mechanisms that contribute to this disease are still poorly understood. Now, Robert Costa and colleagues reveal that the Foxm $1 b$ transcription factor is essential for HCC development and can be inhibited by an Arf peptide.

The authors treated mice with the HCC-inducing chemicals diethylnitrosoamine (DEN) and phenobarbital (PB). In wild-type mice, Foxm $1 \mathrm{~b}$ expression increased in hepatocyte nuclei before tumour formation and remained at high levels in HCCs. Mice with Foxm1bnull hepatocytes failed to develop tumours - although hepatocytes showed some signs of preneoplastic change, they failed to proliferate. So, how does Foxm $1 b$ promote hepatocyte proliferation?

Foxm $1 \mathrm{~b}$ regulates expression of Cdc25b, which activates the cellcycle promoter cyclin-dependent kinase 1 (Cdk1). Cdc25b expression was significantly decreased in DEN-PB-treated Foxm1b-deficient livers. Overexpression of Foxm $1 b$ is also associated with reduced nuclear localization of the Cdk inhibitor Kip1 (also known as p27), which inhibits cell-cycle progression. The authors found that nuclear accumulation of Kip1 was sustained in the DEN-PBtreated Foxm $1 b$-deficient hepatocytes. So, nuclear accumulation of the Cdk inhibitor Kip 1 and reduced levels of Cdc25b caused by loss of Foxm $1 \mathrm{~b}$ prevent hepatocyte proliferation in response to liver damage.

Not only can Foxm $1 b$ inhibit Kip1 activity, but the opposite is also true. Foxm $1 \mathrm{~b}$ activity is known to require interaction with Cdk-cyclin complexes. Coimmunoprecipitation experiments showed that mutating the binding site in Foxm $1 \mathrm{~b}$ of the Cdk-cyclin complex prevented association with Kip1, indicating that Kip1 associates with Cdk-cyclin complexes recruited by Foxm $1 b$. Transcriptional activity of Foxm $1 b$ was reduced in osteosarcoma cells co-transfected with a Kip1 expression vector and wild-type Foxm $1 b$, so this interaction inhibits Foxm $1 \mathrm{~b}$ transcriptional activity.

Expression of the tumour suppressor Arf (also known as p19 in mice) is induced by DEN-PB treatment and the authors found that Arf also interacts with Foxm $1 b$. A peptide containing amino acids 26-44 of Arf that was modified to improve cellular uptake was sufficient to bind Foxm 1b, target it to the nucleolus and inhibit its transcriptional activity. Furthermore, conditional overexpression of Foxm $1 b$ in osteosarcoma cells, which stimulates anchorage-dependent growth in soft agar, was inhibited by this Arf peptide without causing cellular toxicity.

This work shows that Foxm $1 b$ is required for HCC formation and that Arf is an effective therapeutic inhibitor of Foxm $1 b$ function. Whether this strategy will prove useful in preclinical and clinical trials remains to be established.

Emma Croager

Q1) References and links ORIGINAL RESEARCH PAPER Kalinichenko, V. V. et al. Foxm1b transcription factor is essential for development of hepatocellular carcinomas and is negatively regulated by the $\mathrm{p} 19^{\mathrm{ARF}}$ tumor suppressor. Genes Dev. 18, 830-850 (2004) WEB SITE

Rob Costa's lab:

http://www.uic.edu/com/bcmg/costa.html

\section{IN BRIEF}

\section{ONCOGENESIS}

The translation factor elF-4E promotes tumor formation and cooperates with c-Myc in lymphomagenesis.

Ruggero, D. et al. Nature Med. 18 April 2004 (doi:10.1038/nm1042)

The translation factor eIF4E has been implicated in tumorigenesis. Ruggero et al. showed that transgenic expression of eIF4E causes mice to develop B-cell lymphomas and other tumour types. They found that c-Myc cooperates with eIF4E to promote lymphomagenesis, and propose a model in which c-Myc overrides eIF4E-induced cellular senescence and eIF4E antagonizes c-Myc-dependent apoptosis.

\section{DIAGNOSTICS}

\section{A preoperative diagnostic test that distinguishes} benign from malignant thyroid carcinoma based on gene expression.

Cerutti, J. M. et al. J. Clin. Invest. 113, 1234-1242 (2004)

The techniques that are used to biopsy thyroid nodules can not distinguish between benign follicular thyroid adenoma and invasive follicular carcinoma, so all patients with follicular lesions undergo thyroid resection. Cerutti et al. used serial analysis of gene expression to identify genes that were differentially expressed in the two tumour types, and could therefore improve diagnosis. They showed that a combination of genes can be used to identify carcinomas with $83 \%$ accuracy, so this approach might spare patients from unnecessary surgery.

\section{ONCOGENESIS}

Germ-line expression of an oncogenic erbB2 allele confers resistance to erbB2-induced mammary tumorigenesis.

Andrechek, E. R. et al. Proc. Natl Acad. Sci. USA 101, $4984-4989$ (2004)

Mammary-specific expression of an activated ErbB2 allele leads to the formation of mammary adenocarcinomas in mice. Andrechek et al. tested the effect of expressing the same allele in the germline. Despite levels of ErbB2 expression in the mammary epithelium comparable to those of animals with tissue-specific activation, mice carrying germline ErbB2 did not develop tumours. The authors suggest that feedback mechanisms during development compensate for germline oncogene expression.

\section{EPIGENETICS}

Germline epimutation of MLH1 in individuals with multiple cancers.

Suter, C. M. et al. Nature Genet. 4 April 2004 (doi:10.1038/ng1342)

Epigenetic silencing of tumour suppressors can lead to cancer. Suter et al. identified two individuals with histories of colorectal cancer who had soma-wide epigenetic inactivation of the DNA mismatch repair gene $M L H 1$, indicating that germline epimutation can lead to cancer predisposition. Spermatozoa from one of these individuals also carried the $M L H 1$ epimutation, indicating that this type of defect can be transmitted to offspring. 